\title{
A PROOF OF LENS RIGIDITY IN THE CATEGORY OF ANALYTIC METRICS
}

\author{
JAMES VARGO
}

\begin{abstract}
Consider a compact Riemannian manifold with boundary. If all maximally extended geodesics intersect the boundary at both ends, then to each geodesic $\gamma(t)$ we can form the triple $(\dot{\gamma}(0), \dot{\gamma}(T), T)$, consisting of the initial and final vectors of the segment as well as the length between them. The collection of all such triples comprises the lens data. In this paper, it is shown that in the category of analytic Riemannian manifolds, the lens data uniquely determine the metric up to isometry. There are no convexity assumptions on the boundary, and conjugate points are allowed, but with some restriction.
\end{abstract}

\section{An introduction including the result proved}

Let $(M, g)$ be a compact, Riemannian manifold with boundary $\partial M$, and let it be non-trapping. That means all geodesics, when maximally extended, terminate at the boundary at both their ends. Let $S M$ denote its sphere bundle. Then for any vector $v \in \partial S M$, the geodesic $\gamma_{v}$ originating at $v$ eventually leaves the manifold after some distance $T$. Let $\ell(v)$ denote the length of the geodesic, and let $\Sigma(v)=\dot{\gamma}_{v}(T)$ denote its terminal vector.

$$
\Sigma: \partial S M \rightarrow \partial S M
$$

is called the scattering map. Together, $\Sigma$ and $\ell$ comprise the lens data of $(M, g)$.

The lens rigidity conjecture states that one may recover a Riemannian manifold up to isometry from its lens data $(\Sigma, \ell)$. To be more precise, suppose we have two non-trapping Riemannian manifolds $\left(M_{i}, g_{i}\right), i=1,2$ which share the same boundary. That is, $\partial M_{1}=\partial M_{2}$ (henceforth both will be denoted $\partial M$ ). Then for any point $x \in \partial M$, there is a natural correspondence

$$
\Lambda_{x}: \partial S_{x} M_{1} \rightarrow \partial S_{x} M_{2} .
$$

Indeed, a unit vector at the boundary of a Riemannian manifold is uniquely characterized by its inward normal component and the direction of its tangential projection. So we shall say that $v_{2}=\Lambda\left(v_{1}\right)$ if these two quantities agree for $v_{1}$ and $v_{2}$, respectively.

Definition 1. Let $\left(\Sigma_{i}, \ell_{i}\right)$ denote the lens data for the manifold $\left(M_{i}, g_{i}\right): i=1,2$. We shall say that the two manifolds have the same lens data if $\Lambda \circ \Sigma_{1}=\Sigma_{2} \circ \Lambda$ and $\ell_{1}=\ell_{2} \circ \Lambda$.

Conjecture 1. If $\left(M_{1}, g_{1}\right)$ and $\left(M_{2}, g_{2}\right)$ are non-trapping with the same lens data, then the two manifolds are related by an isometry that fixes the points of the boundary $\partial M$. That is, there exists a diffeomorphism $\varphi: M_{1} \rightarrow M_{2}$ satisfying $\left.\varphi\right|_{\partial M}=i d$ and $\varphi^{*} g_{2}=g_{1}$.

Received by the editors December 5, 2008. 
The lens rigidity problem is a generalization of the boundary rigidity problem. In that problem, the initial data is taken to be the boundary distance function

$$
\rho_{g}: \partial M \times \partial M \rightarrow \mathbb{R} \text {. }
$$

$\rho_{g}(x, y)$ is equal to the length of the shortest curve joining $x$ to $y$. Of course, metrics related by an isometry fixing the boundary will also yield the same boundary distance function. The boundary rigidity problem is whether this is the only obstruction to unique recovery of $g$ from $\rho_{g}$.

If a metric $g$ has the property that the only other metrics with the same boundary distance function are isometric to $g$, then $g$ is called boundary rigid. There are many examples of metrics that are not boundary rigid. Indeed, $\rho_{g}$ only records the lengths of the shortest paths, and it is not hard to construct metrics for which the shortest paths do not enter certain open subsets of the manifold. To circumvent this problem, the assumption of simplicity is usually made on the metric.

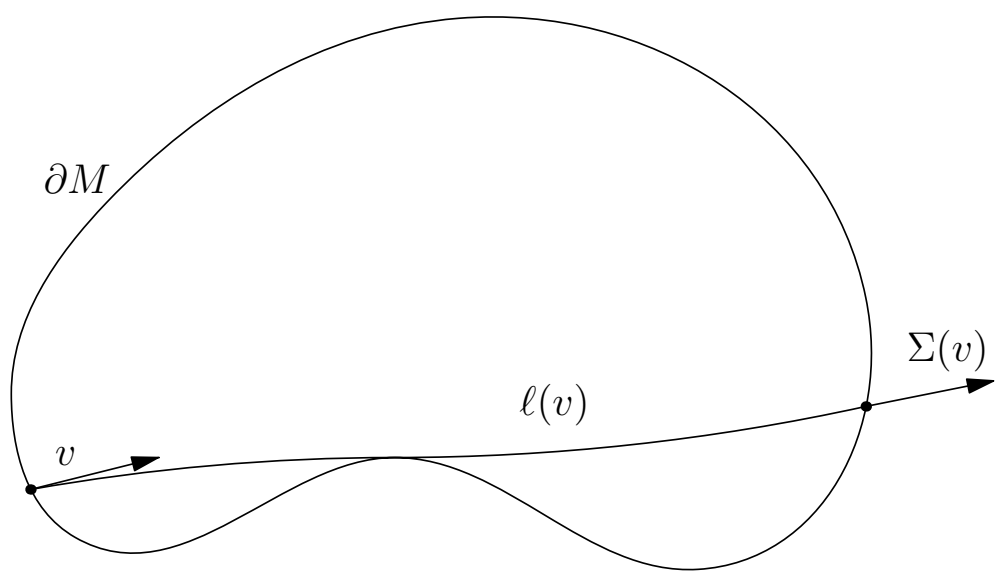

Figure 1. The Lens Data

Definition 2. The Riemannian manifold $(M, g)$ is simple, if $\partial M$ is strictly convex with respect to $g$, and for any $x \in M$, the exponential map $\exp _{x}: \exp _{x}^{-1}(M) \rightarrow M$ is a diffeomorphism.

A simple manifold has the property of being geodesically convex. That is, every pair of points is connected by a unique geodesic and that geodesic is length minimizing. Topologically, a simple manifold is a ball. Michel [9] was the first to conjecture that simple Riemannian manifolds are boundary rigid. This has been proved recently in two dimensions [10]. It has also been proved for subdomains of Euclidean space [7], for metrics close to the Euclidean [2], and symmetric spaces of negative curvature [1]. In [13], Stefanov and Uhlmann proved a local boundary rigidity result. If $g$ belongs to a certain generic set which includes real-analytic metrics, and $g^{\prime}$ is sufficiently close to $g$, then $\rho_{g}=\rho_{g^{\prime}}$ implies that $g$ and $g^{\prime}$ be isometric. For other local results see [4], $[6],[8],[12]$. It is shown in [11] that the lens rigidity problem is equivalent to the boundary rigidity problem if the manifold is simple. 
If the manifold is not simple, the lens data carries more information than the boundary distance function. Indeed, it includes the lengths of all geodesics, so in the case that $g$ be non-trapping, these geodesics pass through every point of the manifold in every direction. However, if the manifold is trapping, there are examples in which the lens data is not sufficient to determine the metric, (see [5]). There are not many results on the lens rigidity problem, but the following are notable. If a manifold is lens rigid a finite quotient of it is also lens rigid [3]. In [11], Stefanov and Uhlmann generalized their local result for simple metrics to obtain a local lens rigidity result. There are some assumptions on conjugate points and a topological assumption. Assuming these, if $g$ belongs to a certain generic set which includes realanalytic metrics, and $g^{\prime}$ is another metric with the same lens data that, a-priori, is known to be sufficiently close to $g$, then $g^{\prime}$ is isometric to $g$.

In this paper, the following statement is proved.

Theorem 1. Let $\left(M_{i}, g_{i}\right), i=1,2$ be non-trapping analytic Riemannian manifolds with a common, analytic boundary $\partial M$. Further, assume that in each connected component of $S\left(\partial M_{1}\right)$, there exists $\left(x_{0}, \xi_{0}\right)$ such that $x_{0}$ is not conjugate to any points of $\partial M$ that lie along the geodesic $\gamma_{x_{0}, \xi_{0}}$. Then if the two manifolds have the same lens data, there must exist an analytic diffeomorphism $\varphi: M_{1} \rightarrow M_{2}$ with $\left.\varphi\right|_{\partial M}=i d$ and $\varphi^{*} g_{2}=g_{1}$.

Note the slightly asymmetric nature of the second hypothesis. This property is used in the proof of Theorem 2 to guarantee the possibility of a certain construction on the lens data. Since $g_{2}$ has the same lens data, the same construction will work automatically, even though, a priori, there is no reason why the condition of the theorem should also be true for $g_{2}$.

\section{Constructing an isometry on a band about the boundary}

Let $(M, g)$ be a general compact Riemannian manifold with boundary. Let $\nu$ be the field of inward unit normal vectors at the boundary $\partial M$, and let $x_{0} \in \partial M$ be a boundary point. Then there is a small neighborhood $N \subset \partial M$ of $x_{0}$ and a number $\epsilon>0$ such that the mapping

$$
\exp _{\nu}: N \times[0, \epsilon) \rightarrow M
$$

given by $\left(x^{\prime}, x^{n}\right) \mapsto \exp _{x^{\prime}}\left(x^{n} \nu\right)$ gives a local coordinate system. These are the boundary normal coordinates. Through them, the metric has the form

$$
d s^{2}=g_{\alpha \beta} d x^{\prime \alpha} d x^{\prime \beta}+\left(d x^{n}\right)^{2},
$$

where $\alpha, \beta$ are indices running over the tangential directions. Now let $\bar{M}$ be an open manifold slightly extending $M$ and extend $g$ smoothly to $\bar{M}$ (extend by analytic continuation in the case that $(M, g)$ is analytic). By choosing a smaller $\epsilon$ if necessary, we may now use our boundary normal coordinates as a coordinate system in $\bar{M}$ by allowing the coordinate $x^{n}$ to vary over the set $(-\epsilon, \epsilon)$.

By compactness, we may choose $\epsilon$ uniformly over the whole boundary. We may also select $\epsilon$ sufficiently small so that our boundary normal coordinates give a global diffeomorphism

$$
\exp _{\nu}: \partial M \times(-\epsilon, \epsilon) \rightarrow V
$$

where $V$ is a neighborhood of $\partial M$ in $\bar{M}$. 
To show this, it is only necessary to prove the above mapping injective. Around each point of $\partial M$, let $N$ be a connected open neighborhood such that $\exp _{\nu}$ defined on $N \times(-\epsilon, \epsilon)$ is injective. By compactness, $\partial M$ is covered by a finite number of such neighborhoods $N_{1}, \ldots, N_{m}$. There exists a number $\delta>0$ such that for any two points $x, y \in \partial M, x, y$ must belong to a common neighborhood $N_{i}$ if $d(x, y)<\delta$. Take $\epsilon$ to be less than half of $\delta$.

Suppose there are points $x, y \in \partial M$ and numbers $s, t \in(-\epsilon, \epsilon)$ such that

$$
\exp _{\nu}(x, s)=\exp _{\nu}(y, t)
$$

Then by the triangle inequality, $d(x, y)<s+t<\delta$ which shows that $x, y$ belong to a common neighborhood $N_{i}$. But on $N_{i} \times(-\epsilon, \epsilon), \exp _{\nu}$ is injective. Hence $x=y, s=t$.

We define the manifold $\tilde{M}$ to be $M \cup U$, where $U$ is a collar defined by:

$$
U=\left\{x:-\epsilon \leq x^{n} \leq 0\right\} .
$$

Next, note that the set $V$ is a subset of $\tilde{M}$, and can be described as the set of points in $\tilde{M}$ whose distance from $\partial M$ is less than $\epsilon$ :

$$
V=\{x \in \tilde{M}: d(x, \partial M)<\epsilon\}
$$

See Figure 2

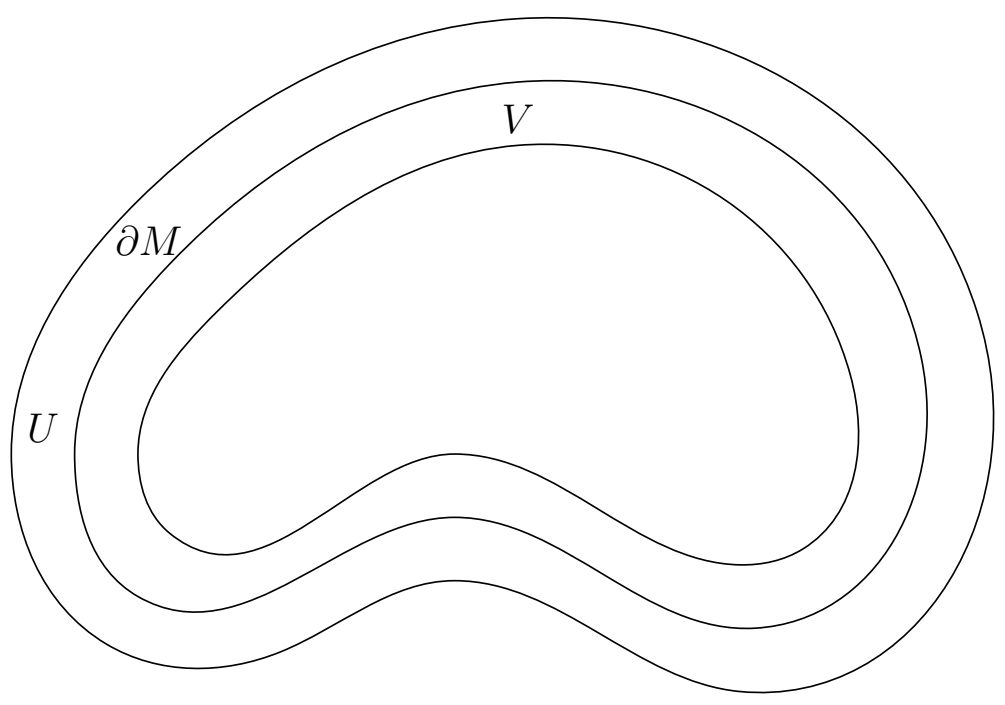

FigurE 2. $\tilde{M}$

Theorem 1 relies principally on the following theorem proved by Stefanov and Uhlmann in [11].

Theorem 2. Let $(M, g)$ be a compact Riemannian manifold with boundary. Let $\left(x_{0}, \xi_{0}\right) \in S(\partial M)$ be such that the maximal geodesic $\gamma_{x_{0}, \xi_{0}}$ through it is of finite length, and assume that $x_{0}$ is not conjugate to any point in $\gamma_{x_{0}, \xi_{0}} \cap \partial M$. Then the jet of $g$ at $x_{0}$ in boundary normal coordinates is uniquely determined by the lens data $(\Sigma, \ell)$. 
Corollary 1. Assume $(M, g)$ is analytic with analytic boundary and that, in each connected component of $S(\partial M)$, there is at least one vector $\left(x_{0}, \xi_{0}\right)$ satisfying the conditions of the theorem. Then the lens data uniquely determine the metric $g$ in boundary normal coordinates.

Proof. As above we let $V$ denote the set of points $\{x \in \tilde{M}: d(x, \partial M) \leq \epsilon\}$. Then by hypothesis, in each component of $V$ there is at least one point at which the jet of the metric is determined. Since the metric is analytic, it must be uniquely determined on all of $V$.

Now we apply this to our two Riemannian manifolds $\left(M_{i}, g_{i}\right)$, taking an $\epsilon$ sufficiently small to work for both. We obtain $\exp _{\nu_{i}}: \partial M \times[-\epsilon, \epsilon] \rightarrow V_{i} \subset \tilde{M}_{i}$. Using these coordinates, both metrics have the form $g_{\alpha \beta} d x^{\prime \alpha} d x^{\prime \beta}+\left(d x^{n}\right)^{2}$. By the corollary, the functions $g_{\alpha \beta}$ coincide for the two metrics throughout the domains $\partial M \times[-\epsilon, \epsilon]$, which means that the mapping $\varphi_{0}: V_{1} \rightarrow V_{2}$ defined by $\varphi_{0}=\exp _{\nu_{2}} \circ \exp _{\nu_{1}}^{-1}$ is an isometry.

Note that $\left.\varphi_{0}\right|_{\partial M}=i d$, and $\varphi_{0 *}\left(\nu_{1}\right)=\nu_{2}$. In particular, $\varphi_{0 *}$ must preserve directions in $T \partial M$ and must preserve components in the normal direction. Thus $\left.\varphi_{0 *}\right|_{S M_{1}}=\Lambda$, the mapping that relates the lens data of our two manifolds.

\section{Extension of the isometry to the entire manifold}

The rest of this paper shall be concerned with extending $\varphi_{0}$ to an isometry $\varphi$ : $\tilde{M}_{1} \rightarrow \tilde{M}_{2}$. If the extension exists, then it must be uniquely defined. Indeed, given a point $x_{0} \in U_{1}$ and a unit vector $\xi$ at $x_{0}$, we must require

See Figure 3.

$$
\varphi\left(\exp _{x_{0}}^{g_{1}}(t \xi)\right)=\exp _{\varphi_{0}\left(x_{0}\right)}^{g_{2}}\left(t \varphi_{0 *} \xi\right)
$$

By the non-trapping assumption, all points $x \in M_{1}$ lie on a geodesic originating in $U_{1}$. Therefore this equation uniquely determines the extended mapping $\varphi$. However, it is not at all clear that the equation yields a well-defined mapping. To get around this problem, we shall first define a mapping $\tilde{\varphi}: S M_{1} \rightarrow M_{2}$ and then show that the values of $\tilde{\varphi}$ only depend on the basepoint $x \in M_{1}$.

Choose $(x, v) \in S M_{1}$, and consider the geodesic $\gamma_{x,-v}$ (note the reversal of $v$ ). Let $T_{0}=T_{0}(v) \geq 0$ be the first time at which this curve first leaves $M_{1}$ and enters $U_{1}$. That is,

$$
T_{0}=T_{0}(v)=\inf \left\{t \geq 0: \gamma_{x,-v}(t) \notin M_{1}\right\} .
$$

This value exists because of the nontrapping assumption. Similarly, we define $T_{1}=$ $T_{1}(v)$ to be the first time after $T_{0}(v)$ at which the curve leaves the interior of $U_{1}$ :

$$
T_{1}=T_{1}(v)=\inf \left\{t>T_{0}(v): \gamma_{x,-v}(t) \in \partial U_{1}\right\} .
$$

$\left(M_{1}, g_{1}\right)$ is assumed non-trapping, however, we make no assumptions on whether $M_{1} \cup U_{1}$ is trapping, so if, somehow, the curve never leaves the interior of $U_{1}$, we set $T_{1}=\infty$.

We claim that $T_{1}>T_{0}$. This follows from the assumption that $\partial M$ and the metric are both analytic. Consequently, a geodesic cannot have contact of infinite order 


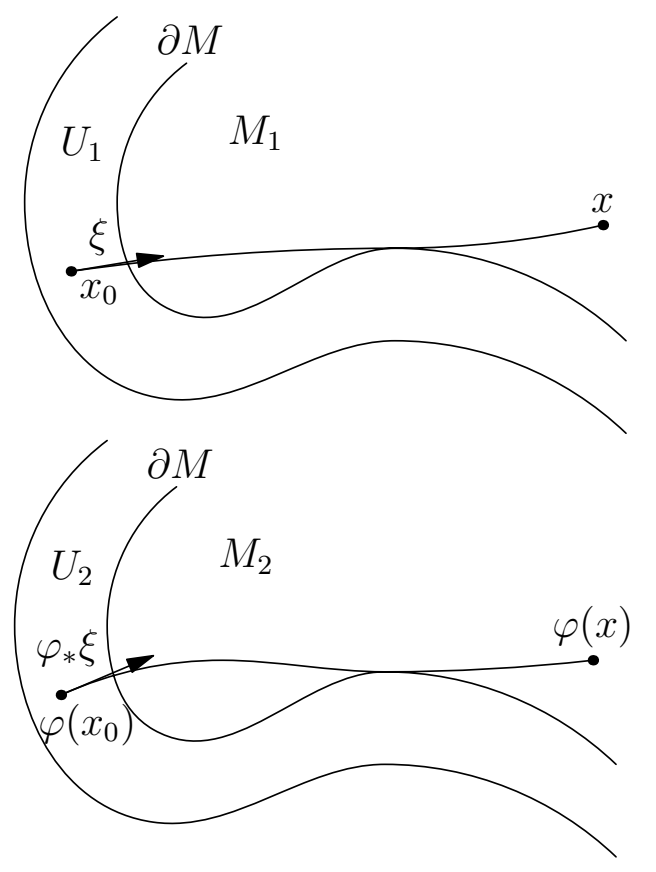

FiguRE 3

with the boundary without being trapped in the boundary. Therefore, at $t=T_{0}$, we conclude that there exists $m \geq 1$ for which

$$
\begin{aligned}
& \partial_{t}^{k}\left(x^{n} \circ \gamma_{x,-v}\right)\left(T_{0}\right)=0, \quad 0 \leq k<m ; \\
& \partial_{t}^{m}\left(x^{n} \circ \gamma_{x,-v}\right)\left(T_{0}\right)<0 .
\end{aligned}
$$

This shows that for some small amount of time after $T_{0}$, the geodesic must remain entirely outside $M_{1}$. Hence $T_{1}>T_{0}$.

Now we let $T=T(v)$ be an arbitrarily chosen number strictly between $T_{0}$ and $T_{1}$, and we let $\xi_{v}=-\dot{\gamma}_{x,-v}(T)$. By construction, $x=\exp ^{g_{1}}\left(T \xi_{v}\right)$. We define $\tilde{\varphi}(x, v)$ by:

\section{See Figure 4.}

$$
\tilde{\varphi}(x, v)=\exp ^{g_{2}}\left(T \varphi_{0 *} \xi_{v}\right)
$$

Proposition 1. $\tilde{\varphi}(x, v)$ is a well defined function on $S M_{1}$ with values in $M_{2}$.

Proof. We must show two things: first, that $\exp ^{g_{2}}\left(T \varphi_{0 *} \xi_{v}\right)$ is a point in $M_{2}$; second, that the value of $\tilde{\varphi}$ is independent of the choice of $T$.

The curve $\gamma_{\xi_{v}}(t)=\exp ^{g_{1}}\left(t \xi_{v}\right): 0 \leq t \leq T$ is composed of two segments; the first of which lies within $U_{1}$, the second of which lies within $M_{1}$. The break between the two occurs at $t=T-T_{0}$. We conclude that $\ell\left(\dot{\gamma}_{\xi_{v}}\left(T-T_{0}\right)\right) \geq T_{0}$.

The curve $\exp ^{g_{2}}\left(t \varphi_{0 *} \xi_{v}\right): t \in[0, T]$ is also composed of two segments of length $T-T_{0}$ and $T_{0}$ lying in $U_{2}$ and $M_{2}$ respectively. Indeed, for $t \in\left[0, T-T_{0}\right]$, we have

$$
\exp ^{g_{2}}\left(t \varphi_{0 *} \xi_{v}\right)=\varphi_{0}\left(\exp ^{g_{1}}\left(t \xi_{v}\right)\right)
$$

from the fact that $\varphi_{0}$ is an isometry on $U_{1}$. Hence the left side belongs to $U_{2}$. 


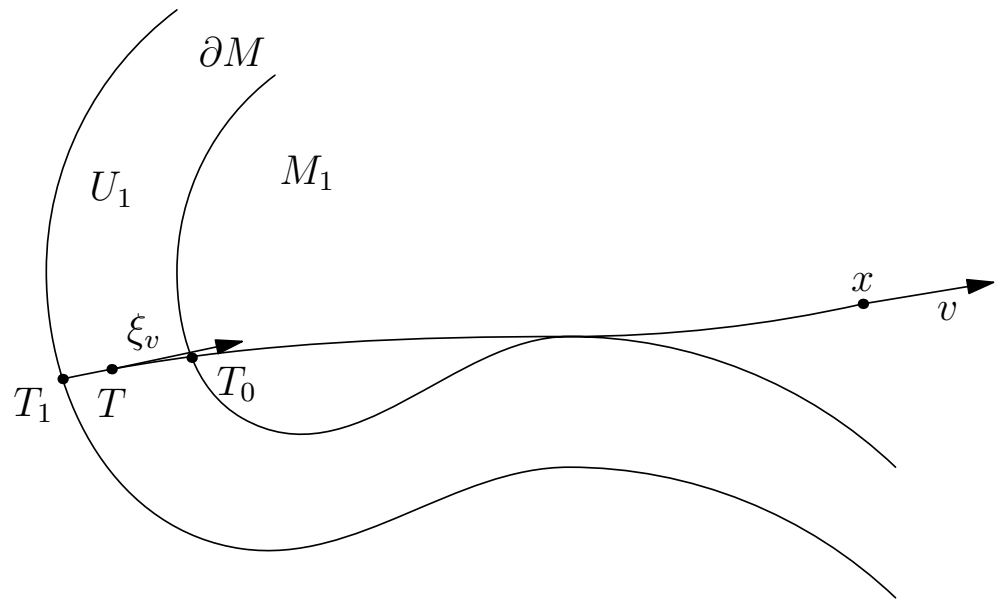

Figure 4. The Construction of $\tilde{\varphi}$

To show that the remainder of the curve $\exp ^{g_{2}}\left(t \varphi_{0 *} \xi_{v}\right)$ lies in $M_{2}$, we look at the lens data. If we differentiate at $t=T-T_{0}$, we obtain from equation (1), $\varphi_{0 *}\left(\dot{\gamma}_{\xi_{v}}\left(T-T_{0}\right)\right)$ which equals $\Lambda\left(\dot{\gamma}_{\xi_{v}}\left(T-T_{0}\right)\right)$.

By the fact that $M_{1}$ and $M_{2}$ have the same lens data, we conclude that

$$
\ell\left(\varphi_{0 *}\left(\dot{\gamma}_{\xi_{v}}\left(T-T_{0}\right)\right)\right) \geq T_{0},
$$

so the point $\exp \left(T \varphi_{0 *} \xi_{v}\right)$ does indeed exist and lie in $M_{2}$.

Now let $T^{\prime}$ be another time in between $T_{0}$ and $T_{1}$, and let $\xi_{v}^{\prime}$ be the corresponding vector. Without loss of generality we may assume that $\Delta T=T-T^{\prime}>0$. Then we have the following identity

$$
\exp ^{g_{1}}\left(t \xi_{v}\right)=\exp ^{g_{1}}\left((t-\Delta T) \xi_{v}^{\prime}\right) .
$$

By the definitions of $T_{0}$ and $T_{1}$, the curve $\exp ^{g_{1}}\left(t \xi_{v}\right): 0 \leq t \leq \Delta T$ is a geodesic segment lying entirely within the interior of $U_{1}$. Since $\varphi_{0}$ is an isometry on $U_{1}$, the vectors $\varphi_{0 *} \xi_{v}$ and $\varphi_{0 *} \xi_{v}^{\prime}$ must also be tangent to a common geodesic at a distance of $\Delta T$. Hence

$$
\exp ^{g_{2}}\left(t \varphi_{0 *} \xi_{v}\right)=\exp ^{g_{2}}\left((t-\Delta T) \varphi_{0 *} \xi_{v}^{\prime}\right) .
$$

Setting $t=T$, we obtain the needed result.

Proposition 2. For fixed $x_{0}, \tilde{\varphi}\left(x_{0}, v\right)$ is constant.

Proof. The strategy here is to prove that $\tilde{\varphi}\left(x_{0}, v\right)$ is locally constant. Then the statement follows from the connectedness of the sphere. First, we need a lemma.

For a pair of points in $\tilde{M}$, let $d(x, y)$ denote the distance between them. This function is not necessarily smooth, even off the diagonal. However, the next lemma shows that the squared distance function $d(x, y)^{2}$ is as smooth as the metric for $(x, y)$ sufficiently close to each other. 
Lemma 1. Let $\tilde{M}$ be as above (with subscript omitted). For every $x_{0}$ in the interior of $\tilde{M}$, there exists a positive number $r$ such that the squared distance function is analytic on the set

$$
\Delta_{r}\left(x_{0}\right)=\left\{(x, y): d\left(x, x_{0}\right)<r, d(x, y)<r\right\} .
$$

If $K$ is a compact set contained within the interior of $\tilde{M}$, then there is an open $O \subset \tilde{M}$ containing $K$ and a positive number $r$ such that the squared distance function is analytic on the set

$$
\Delta_{O, r}(K)=\{(x, y): x \in O, d(x, y)<r\} .
$$

Proof. We choose $r>0$ so that the ball $B_{2 r}\left(x_{0}\right)$ is contained within $\tilde{M}$ and is geodesically convex (see [14], Theorem 6.2, noting that the restriction on the radius is only that it be sufficiently small). By definition every pair of points in $B_{2 r}\left(x_{0}\right)$ is joined by a unique geodesic segment contained entirely within $B_{2 r}\left(x_{0}\right)$. Moreover, that segment is length-minimizing.

Now assume that $(x, y) \in \Delta_{r}\left(x_{0}\right)$. Then there is exactly one geodesic segment connecting them whose length is less than $r$. Indeed, there is at least one, since the two points lie within $B_{2 r}\left(x_{0}\right)$. If there were another geodesic segment connecting them, it would have to leave $B_{2 r}\left(x_{0}\right)$ at some point. Since $d\left(x_{0}, x\right)<r$, such a segment would necessarily have length greater than $r$.

This shows that the mapping

$$
\left\{(x, v): d\left(x, x_{0}\right)<r,|v|_{g}<r\right\} \rightarrow \Delta_{r}\left(x_{0}\right)
$$

given by $(x, v) \mapsto\left(x, \exp _{x}(v)\right)$ is bijective. Naturally, the exponential map is analytic as long as the metric is analytic. By the inverse function theorem, $\exp ^{g}$ gives a diffeomorphism between these two sets. Through this diffeomorphism, the squared distance function is expressed $d(x, y)^{2}=g_{i j} v^{i} v^{j}$, which is analytic as long as $g$ is analytic.

The second statement of the lemma follows from the first by a compactness argument. Indeed, for every $x_{0} \in K$ we take the number $r$ from the first statement and form the ball $B_{r}\left(x_{0}\right)$. All such balls form an open cover of $K$. We take a finite subcover, let $O$ be the union of its members, and let $r$ be the smallest radius in that subcover.

Now fix a vector $\left(x_{0}, v\right)$ and choose $T=T(v)$ and $\xi_{v}$. Let $y_{0} \in U_{1}$ be the basepoint of the vector $\xi_{v}$ so that $x_{0}=\exp _{y_{0}}^{g_{1}}\left(T \xi_{v}\right)$. Also, let $\gamma_{1}=\gamma_{x_{0} y_{0}}$ denote the geodesic segment connecting the two points. Let $O_{1}, r_{1}$ be the open set and radius corresponding to the compact set $\gamma_{1}$ as in the lemma.

In $\tilde{M}_{2}$, we have a corresponding segment $\gamma_{2}$ between the points $\varphi_{0}\left(y_{0}\right)$ and $\tilde{\varphi}\left(x_{0}, v\right)$. It is given by the curve

$$
\exp ^{g_{2}}\left(t \varphi_{0 *} \xi_{v}\right):, 0 \leq t \leq T .
$$

Let $r_{2}$ be the radius corresponding to $\gamma_{2}$ as in the lemma.

Let $r$ denote the positive number

$$
r=\inf \left\{d\left(\gamma_{1}, \partial \tilde{M}_{1}\right), d\left(y_{0}, \partial U_{1}\right), r_{1}, r_{2}\right\} .
$$


By continuity, there exists a neighborhood $N$ of $v$ in $S_{x_{0}} M_{1}$ sufficiently small such that for all $w \in N$,

$$
d\left(\gamma_{x_{0},-v}(t), \gamma_{x_{0},-w}(t)\right)<r
$$

for all $t$ in the interval $[0, T]$. The restrictions on $r$ guarantee that the curve $\gamma_{x_{0},-w}(t)$ remain within $\tilde{M}_{1}$ and that its endpoint, $\gamma_{x_{0},-w}(T)$, be in the interior of $U_{1}$. For each $w$, let $\eta_{w}=-\dot{\gamma}_{x_{0},-w}(T)$. We prove $\tilde{\varphi}\left(x_{0}, v\right)=\tilde{\varphi}\left(x_{0}, w\right)$ by breaking this into the two equations:

$$
\begin{gathered}
\exp ^{g_{2}}\left(T \varphi_{0 *} \xi_{v}\right)=\exp ^{g_{2}}\left(T \varphi_{0 *} \eta_{w}\right) \\
\exp ^{g_{2}}\left(T \varphi_{0 *} \eta_{w}\right)=\exp ^{g_{2}}\left(T(w) \varphi_{0 *} \xi_{w}\right)
\end{gathered}
$$

See Figure 5.

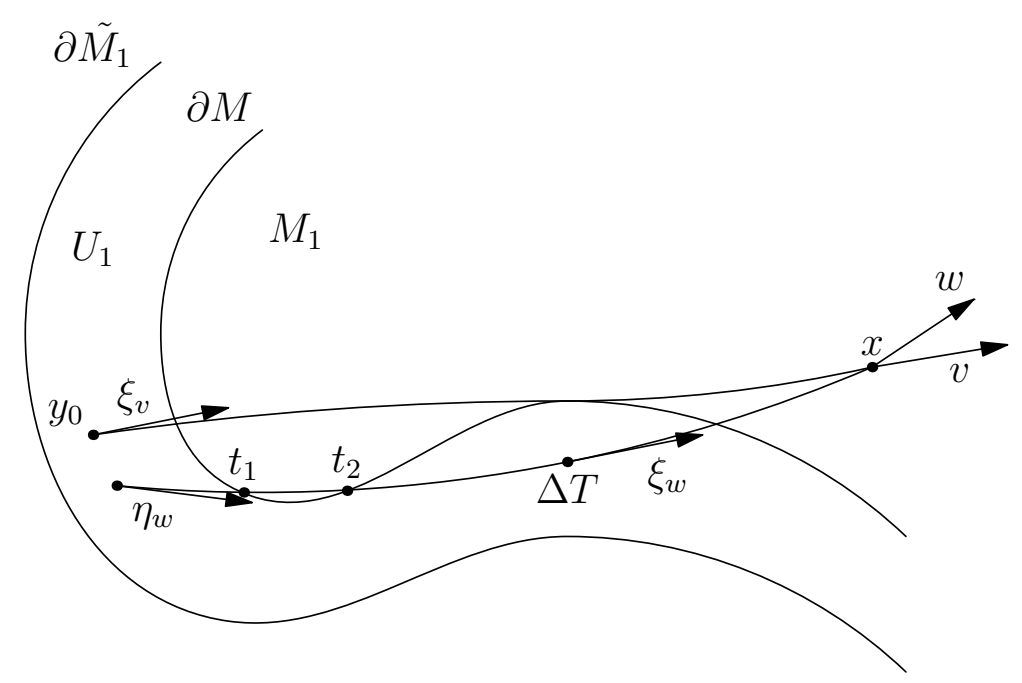

FiguRE 5

Consider the function

$$
\rho_{1}(t)=d_{g_{1}}^{2}\left(\exp \left(t \xi_{v}\right), \exp \left(t \eta_{w}\right)\right) .
$$

By our choices of $r$ and $N$, and by the lemma, this is an analytic function for $t \in[0, T]$.

Now we consider $\tilde{M}_{2}$, and define

$$
\rho_{2}(t)=d_{g_{2}}^{2}\left(\exp ^{g_{2}}\left(t \varphi_{0 *} \xi_{v}\right), \exp ^{g_{2}}\left(t \varphi_{0 *} \eta_{w}\right)\right) .
$$

Since $\left.\varphi_{0}\right|_{U_{1}}: U_{1} \rightarrow U_{2}$ is an isometry, the functions $\rho_{1}$ and $\rho_{2}$ must coincide for small values of $t$. Also, we note that the function $\rho_{2}(t)$ is analytic as long as $d_{g_{2}}\left(\exp ^{g_{2}}\left(t \varphi_{0 *} \xi_{v}\right), \exp ^{g_{2}}\left(t \varphi_{0 *} \eta_{w}\right)\right)<r$, since $r$ was chosen to be smaller than $r_{2}$. Therefore, by analytic continuation, the functions $\rho_{1}$ and $\rho_{2}$ are equal up to the first point $t_{0}$ where $\rho_{2}=r^{2}$. But by continuity, we would then have $\rho_{1}\left(t_{0}\right)=r^{2}$, which does not occur. Therefore, we see that $\rho_{1}(t)=\rho_{2}(t)$ throughout the interval $0 \leq t \leq T$. In particular, we find that $\rho_{2}(T)=0$, which verifies equation (2).

If $T$ lies between $T_{0}(w)$ and $T_{1}(w)$, then equation (3) is nothing but a restatement that $\tilde{\varphi}\left(x_{0}, w\right)$ is well defined. Clearly, $T>T_{0}(w)$, so assume that it is also greater than 
$T_{1}(w)$ (The possiblity that $T=T_{1}(w)$ is ruled out by the fact that $\gamma_{x,-w}\left(T_{1}(w)\right) \in$ $\partial M)$. Fix a number $T^{\prime}=T(w)$, and a corresponding $\xi_{w}$. If we let $\Delta T=T-T^{\prime}$, then we have the equation

$$
\exp ^{g_{1}}\left(t \eta_{w}\right)=\exp ^{g_{1}}\left((t-\Delta T) \xi_{w}\right) .
$$

Let $\gamma(t)=\exp ^{g_{1}}\left(t \eta_{w}\right)$, for $0 \leq t \leq \Delta T$. If it happens that it lies entirely within $U_{1}$, then the same proof that we used to show that $\tilde{\varphi}$ is well-defined will verify equation (3). So assume that $\gamma(t)$ does not lie entirely within $U_{1}$. Then we can uniquely partition it into subsegments which alternately lie in $U_{1}$ and $M_{1}$. Indeed, we define

$$
\begin{array}{cc}
t_{0}= & 0, \\
t_{1}= & \inf \left\{t>0: \gamma(t) \notin U_{1}\right\}, \\
t_{2}= & \inf \left\{t>t_{1}: \gamma(t) \notin M_{1}\right\}, \\
\vdots & \vdots \\
t_{m-1}= & \inf \left\{t>t_{m-2}: \gamma(t) \notin M_{1}\right\}, \\
t_{m}= & \Delta T .
\end{array}
$$

The fact that $0=t_{0}<t_{1}<\ldots<t_{m}=\Delta T$ is true follows from the same reasoning that was used above to prove that $T_{1}(v)>T_{0}(v)$. The fact that this partition is finite also follows from the analytic assumption. Indeed an analytic curve segment cannot intersect the analytic $\partial M$ more than a finite number of times without being entirely contained within it. Note that the segment $\left.\gamma\right|_{\left[t_{k}, t_{k+1}\right]}$ lies in $U_{1}$ or $M_{1}$ according to whether $k$ is even or odd, respectively. In particular, $m$ is odd.

To prove equation (3), we will use induction to show that for all $k=1,2, \ldots, m$, and all $t \in[0, T]$,

$$
\exp ^{g_{2}}\left(t \varphi_{0 *} \dot{\gamma}(0)\right)=\exp ^{g_{2}}\left(\left(t-t_{k}\right) \varphi_{0 *} \dot{\gamma}\left(t_{k}\right)\right) .
$$

Then setting $k=m$ and $t=T$ yields the result.

Step 1: $\eta_{w}=\dot{\gamma}(0)$ and $\dot{\gamma}\left(t_{1}\right)$ lie on the geodesic $\gamma$ at a distance of $t_{1}$ from each other. Since this segment lies within $U_{1}$ and since $\varphi_{0}$ is an isometry of $U_{1}$ to $U_{2}$, we see that $\varphi_{0 *} \dot{\gamma}(0)$ and $\varphi_{0 *} \dot{\gamma}\left(t_{1}\right)$ also lie on a common geodesic at the same distance apart. Hence equation (4) is established for $k=1$.

Step 2: The next segment of $\gamma$ lies within $M_{1}$. Indeed we have the following:

$$
\dot{\gamma}\left(t_{2}\right)=\Sigma_{g_{1}}\left(\dot{\gamma}\left(t_{1}\right)\right), \ell_{g_{1}}\left(\dot{\gamma}\left(t_{1}\right)\right)=t_{2}-t_{1} .
$$

Since $M_{2}$ has the same lens data as $M_{1}$, we see that $\varphi_{0 *} \dot{\gamma}\left(t_{1}\right)$ and $\varphi_{0 *} \dot{\gamma}\left(t_{2}\right)$ are connected by a geodesic across $M_{2}$ with the same length $t_{2}-t_{1}$. Together with step 1 , this shows that $\varphi_{0 *} \dot{\gamma}\left(t_{2}\right)$ lies tangent to the same geodesic as $\varphi_{0 *} \dot{\gamma}(0)$ at a distance of $t_{2}$. Hence Equation 8 is established for $k=2$.

Step 3: By induction, we may repeat these steps, establishing equation (4) for all $k$ up to $k=m$.

For $x \in M_{1}$, set $\varphi(x)=\tilde{\varphi}(x, v)$. If $x \in V_{1}$, then we are in the domain of the boundary normal coordinates. We choose $v=\frac{\partial}{\partial x^{n}}$. Then $\gamma_{x,-v}$ is the geodesic segment from $x$ to $U_{1}$ normal to $\partial M$. We choose $T(v), \xi_{v}$ so that $x=\exp ^{g_{1}}\left(T(v) \xi_{v}\right)$ and note that the segment $\exp ^{g_{1}}\left(t \xi_{v}\right): 0 \leq t \leq T_{v}$ lies entirely in $V$. We have

$$
\varphi_{0}(x)=\exp \left(T(v) \varphi_{0 *} \xi_{v}\right)=\tilde{\varphi}(x, v)=\varphi(x)
$$


The first equation is true by the fact that $\varphi_{0}$ is an isometry on $V_{1}$. Hence $\varphi$ and $\varphi_{0}$ agree on their common domains. Gluing them together, we form

$$
\varphi: \tilde{M}_{1} \rightarrow \tilde{M}_{2} \text {. }
$$

\section{That $\varphi$ is a diffeomorphism and an isometry}

Let $(x, v)$ lie in the interior of $S_{x} M_{1}$. We choose $T=T(v)$ and $\xi_{v} \in S U_{1}$ so that $\gamma(t)=\exp ^{g_{1}}\left(t \xi_{v}\right)$ is a geodesic in $\tilde{M}_{1}$ that reaches $x$ at time $T$. In the segment $\left[0, T-T_{0}\right], \gamma$ lies entirely within $U_{1}$; whereas on the segment $\left[T-T_{0}, T+\delta\right]$, it lies entirely within $M_{1}$ for $\delta$ sufficiently small. For the first segment, we see that

$$
\varphi(\gamma(t))=\varphi_{0}(\gamma(t))=\exp ^{g_{2}}\left(t \varphi_{0 *} \xi_{v}\right) ;
$$

where the second equality holds by the fact that $\varphi_{0}$ is an isometry on $U_{1}$.

On the second segment, we see that for each pair $(\gamma(t), \dot{\gamma}(t))$, we can choose the same $\xi_{v}$ for $\xi_{\dot{\gamma}(t)}$ with the corresponding $T(\dot{\gamma}(t))=t$. So, for all $t \in[0, T+\delta]$,

$$
\varphi(\gamma(t))=\exp ^{g_{2}}\left(t \varphi_{0 *} \xi_{v}\right) .
$$

This can be rewritten in the form:

$$
\varphi(\gamma(t))=\exp ^{g_{2}}\left(t \varphi_{0 *} \dot{\gamma}(0)\right) .
$$

In fact, the latter equation is true for any geodesic segment $\gamma \mid[0, T]$ that can be partitioned into $\gamma \mid[0, a]$ and $\gamma \mid[a, T]$ with the two subsegments lying entirely in $U_{1}$ and $M_{1}$ respectively.

Proposition 3. $\varphi: \tilde{M}_{1} \rightarrow \tilde{M}_{2}$ is bijective.

Proof. Reversing the roles of $\tilde{M}_{1}$ and $\tilde{M}_{2}$, we can define a mapping $\psi: \tilde{M}_{2} \rightarrow \tilde{M}_{1}$ by the same process by which we defined $\varphi$. In particular it would extend $\varphi_{0}^{-1}$.

The analog to equation (6) is:

$$
\psi(\beta(t))=\exp ^{g_{1}}\left(t \varphi_{0 *}^{-1} \dot{\beta}(0)\right),
$$

where $\beta$ is any geodesic segment composed of two subsegments contained in $U_{2}$ and $M_{2}$, respectively.

Using the notation from above, we would like to prove that $\psi \circ \varphi(\gamma(t))=\gamma(t)$ for $t \in[0, T]$. To that end, we will first show that the geodesic segment $\beta(t)=\varphi(\gamma(t))$ is of the type valid for equation (7).

We note that for $t \in\left[0, T-T_{0}\right], \gamma(t) \in U_{1}$ so $\varphi(\gamma(t))$ must lie in $U_{2}$. For $t \in$ $\left[T-T_{0}, T\right], \gamma(t) \in M_{1}$, so by Proposition $1, \varphi(\gamma(t)) \in M_{2}$.

Therefore, we may apply equation (7), which yields:

$$
\begin{aligned}
\psi \circ \varphi(\gamma(t)) & = & \exp ^{g_{1}}\left(t \varphi_{0 *}^{-1} \dot{\beta}(0)\right) \\
& = & \exp ^{g_{1}}\left(t \varphi_{0 *}^{-1} \varphi_{0 *} \dot{\gamma}(0)\right) \\
& = & \exp ^{g_{1}}(t \dot{\gamma}(0)) \\
& = & \gamma(t) .
\end{aligned}
$$

Since every point in $M_{1}$ lies on some such curve $\gamma(t)$, we conclude that $\psi \circ \varphi=i d$ on $M_{1}$. But we know that the same identity is true on $V_{1}$, so it is true on all of $\tilde{M}_{1}$. By the symmetry of the construction, we conclude $\varphi \circ \psi$ is also the identity.

Proposition 4. $\varphi: \tilde{M}_{1} \rightarrow \tilde{M}_{2}$ is an analytic isometry. 
Proof. Since $\varphi$ is bijective, it is sufficient to prove the statement locally. These properties are already known on $V_{1}$ where $\varphi=\varphi_{0}$, so we assume $x$ lies in the interior $M_{1}$. First we show that all directional derivatives of $\varphi$ exist. Indeed, by differentiating equation (5) at $t=T(v)$, we obtain:

$$
D_{v} \varphi(x)=\partial_{t}(\varphi \circ \gamma)(T(v))=\left.\partial_{t} \exp ^{g_{2}}\left(t \varphi_{0 *} \xi_{v}\right)\right|_{t=T(v)} .
$$

Clearly, the quantity on the right side exists. What's more, it is a vector of length 1. We conclude that $\varphi_{*}$ exists and preserves lengths of vectors. In particular it is nonsingular.

From the fact that it preserves lengths, we derive smoothness. Indeed, $g_{1}=\varphi^{*} g_{2}$, which has the coordinate form:

$$
g_{1}(x)_{i j}=\varphi_{, i}^{k}(x) g_{2}(\varphi(x))_{k j} .
$$

This yields:

$$
g_{1}(x)_{i j} g_{2}(\varphi(x))^{j l}=\varphi_{, i}^{k}(x) .
$$

The left side is once differentiable; hence $\varphi$ is twice differentiable. But then that implies the left side to be twice differentiable which shows $\varphi$ to be three-times differentiable. By an obvious application of induction, $\varphi$ must be smooth.

It only remains to prove that $\varphi$ is analytic. Of course this is already known in $V_{1}$. Since $\varphi$ is a smooth isometry, we can state

$$
\varphi\left(\exp ^{g_{1}} \xi\right)=\exp ^{g_{2}}\left(\varphi_{*} \xi\right)
$$

for any vector $\xi \in T \tilde{M}_{1}$. Given $x_{0}$ in the interior of $M_{1}$, consider a ball of radius $r$, centered at $x_{0}$, which is strictly geodesically convex, and choose any point $y_{0}$ within this ball. Then there is a unique $\xi_{0} \in T_{y} M_{1}$ satisfying $\left|\xi_{0}\right|_{g_{1}}<r$ and $\exp _{y_{0}} \xi_{0}=x$. Moreover, $x_{0}$ and $y_{0}$ are not conjugate along the corresponding geodesic, so

$$
\exp _{y_{0}}^{g_{1}}: \xi \mapsto x
$$

is a local diffeomorphism about $\xi_{0}$. It is analytic by the fact that $g_{1}$ is analytic. Consequently, it's inverse function is analytic. Let $\xi(x)$ denote the inverse, which is defined for $x$ in some neighborhood of $x_{0}$. Then we see that

$$
\varphi(x)=\exp _{\varphi\left(y_{0}\right)}^{g_{2}}\left(\varphi_{*} \xi(x)\right) .
$$

$y_{0}$ is constant, so $\varphi_{*}$ is a constant linear mapping. From the fact that $g_{2}$ is analytic, we see that this mapping is also analytic.

\section{References}

[1] G. Besson, G. Courtois, and S. Gallot, Entropies et rigitités des espaces localement symétriques de courbure strictment négative, Geom. Funct. Anal. 5 (1995) 731-799.

[2] D. Burago and S. Ivanov, Boundary rigidity and filling volume minimality of metrics close to a flat one. To appear.

[3] C. Croke, Boundary and lens rigidity of finite quotients, Proc. AMS 133

[4] C. Croke, N. Dairbekov, and V. Sharafutdinov, Local boundary rigidity of a compact Riemannian manifold with curvature bounded above, Trans. Amer. Math. Soc. 352 (2000) 3937-3956.

[5] C. Croke and B. Kleiner, Conjugacy and rigidity for manifolds with a parallel vector field, J. Diff. Geom. 39 (1994) 659-680.

[6] G. Eskin, Inverse scattering problem in anisotropic media, Comm. Math. Phys. 199 (1998) 471-491.

[7] M. Gromov, Filling Riemannian manifolds, J. Diff. Geom. 39 (1994) 1-148. 
[8] M. Lassas, V. Sharafutdinov, and G. Uhlmann, Semiglobal boundary rigidity for Riemannian metrics, Math. Ann. 325 (2003) 767-793.

[9] R. Michel, Sur la rigidité imposée par la longueur des géodésiques, Invent. Math. 65 (1981) 71-83.

[10] L. Pestov and G. Uhlmann, Two dimensional simple compact manifolds with bondary are boundary rigid, Ann. Math. 161

[11] P. Stefanov and G. Uhlmann, Local lens rigidity with incomplete data for a class of non-simple Riemannian manifolds. To appear in J. Diff. Geom.

[12] — Rigidity for metrics with the same lengths of geodesics, Math. Res. Lett. 5 (1998) 83-96.

[13] — Boundary rigidity and stability for generic simple metrics, J. Amer. Math. Soc. 18 (2005) 975-1003.

[14] S. Sternberg, Lectures on Differential Geometry, Prentice Hall (1964).

Department of Mathematics, University of Washington, Box 354350 Seattle, WA 981954350

E-mail address: jvargo@math.washington.edu 